\title{
ROUGH SINGULAR INTEGRALS WITH KERNELS SUPPORTED BY SUBMANIFOLDS OF FINITE TYPE*
}

\author{
HUSSAIN AL-QASSEM ${ }^{\dagger}$, AHMAD AL-SALMAN $^{\ddagger}$, AND YIBIAO PAN ${ }^{\S}$
}

Key words. $L^{p}$ boundedness, Singular integrals, Fourier transform, block spaces

AMS subject classifications. Primary 42B20; Secondary 42B15, 42B25

1. Introduction. Let $n \geq 2, \mathbf{R}^{n}$ be the $n$-dimensional Euclidean space, and $\mathbf{S}^{n-1}$ denote the unit sphere in $\mathbf{R}^{n}$ equipped with the normalized Lebesgue measure $d \sigma$. For $d \in \mathbf{N}$, let $B(0,1)$ be the unit ball centered at the origin in $\mathbf{R}^{n}$ and $\Phi$ : $B(0,1) \rightarrow \mathbf{R}^{d}$ be a $C^{\infty}$ mapping. Define the singular integral operator $T_{\Phi}$ and the related maximal operator $\mathcal{M}_{\Phi}$ by

$$
\begin{gathered}
T_{\Phi} f(x)=\text { p.v. } \int_{B(0,1)} f(x-\Phi(y)) \frac{\Omega(y)}{|y|^{n}} d y, \\
\mathcal{M}_{\Phi} f(x)=\sup _{0<r \leq 1} \frac{1}{r^{n}} \int_{|y| \leq r}|f(x-\Phi(y))||\Omega(y)| d y
\end{gathered}
$$

for $f \in \mathcal{S}\left(\mathbf{R}^{d}\right)$. Here $\Omega$ is a homogeneous function of degree 0 , integrable over $\mathbf{S}^{n-1}$ and satisfies the vanishing condition

$$
\int_{\mathbf{S}^{n-1}} \Omega(u) d \sigma(u)=0 .
$$

The corresponding maximal truncated singular integral operator $T_{\Phi}^{*}$ is defined by

$$
T_{\Phi}^{*} f(x)=\sup _{\varepsilon>0}\left|\int_{\varepsilon \leq|y|<1} f(x-\Phi(y)) \frac{\Omega(y)}{|y|^{n}} d y\right| .
$$

When $\Phi(y) \equiv y, T_{\Phi}$ is simply the localized version of a classical CalderónZygmund operator and we shall denote it by $T$. Our point of departure is the following $L^{p}$ boundedness result from [St].

TheOrem 1.1. Let $T_{\Phi}$ and $\mathcal{M}_{\Phi}$ be given as in (1.1)-(1.3). Assume that:

(i) $\Phi$ is of finite type at 0 ;

(ii) $\Omega \in C^{1}\left(\mathbf{S}^{n-1}\right)$.

Then for $1<p<\infty$ there exists a constant $C_{p}>0$ such that

$$
\left\|T_{\Phi} f\right\|_{L^{p}\left(\mathbf{R}^{d}\right)} \leq C_{p}\|f\|_{L^{p}\left(\mathbf{R}^{d}\right)}
$$

and

$$
\left\|\mathcal{M}_{\Phi} f\right\|_{L^{p}\left(\mathbf{R}^{d}\right)} \leq C_{p}\|f\|_{L^{p}\left(\mathbf{R}^{d}\right)}
$$

\footnotetext{
*Received November 10, 2003; accepted for publication February 28, 2004.

$\dagger$ Department of Mathematics, Yarmouk University, Irbid-Jordan (husseink@yu.edu.jo).

$\ddagger$ Department of Mathematics, Yarmouk University, Irbid-Jordan (alsalman@yu.edu.jo)

$\S$ Department of Mathematics, University of Pittsburgh, PA 15260, U.S.A. (yibiao@pitt.edu).
} 
for every $f \in L^{p}\left(\mathbf{R}^{d}\right)$.

Recently, the results in Theorem 1.1 were improved by Fan, Guo, and Pan in [FGP] who showed that the $L^{p}$ boundedness of $T_{\Phi}$ and $\mathcal{M}_{\Phi}$ continues to hold if the condition $\Omega \in C^{1}\left(\mathbf{S}^{n-1}\right)$ is replaced by the weaker condition $\Omega \in L^{q}\left(\mathbf{S}^{n-1}\right)$ for some $q>1$. Also, the authors of [FGP] were able to establish the $L^{p}$ boundedness of the maximal operator $T_{\Phi}^{*}$ under the condition $\Omega \in L^{q}\left(\mathbf{S}^{n-1}\right)$ for some $q>1$.

The main purpose of this paper is to present further improvements of the above results in which the condition $\Omega \in L^{q}\left(\mathbf{S}^{n-1}\right)$ is replaced by a weaker condition $\Omega \in$ $B_{q}^{0,0}\left(\mathbf{S}^{n-1}\right)$. It is worth pointing out that the authors of this paper were able in [AqAsP] to show that the condition $\Omega \in B_{q}^{0,0}\left(\mathbf{S}^{n-1}\right)$ is the best possible for the $L^{p}$ boundedness of the classical operator $T$ to hold. Namely, the $L^{p}$ boundedness of $T$ may fail for any $p$ if it is replaced by a weaker condition $\Omega \in B_{q}^{0, v}\left(\mathbf{S}^{n-1}\right)$ for any $-1<v<0$ and $q>1$. The definition of the block spaces $B_{q}^{0, v}\left(\mathbf{S}^{n-1}\right)$ on the sphere will be recalled in Section 2 .

Our main results can be stated as follows.

Theorem 1.2. Let $T_{\Phi}$ and $\mathcal{M}_{\Phi}$ be given as in (1.1)-(1.3). Assume that:

(i) $\Phi$ is of finite type at 0 ;

(ii) $\Omega \in B_{q}^{0,0}\left(\mathbf{S}^{n-1}\right)$ for some $q>1$.

Then

$$
\left\|T_{\Phi} f\right\|_{L^{p}\left(\mathbf{R}^{d}\right)} \leq C_{p}\|f\|_{L^{p}\left(\mathbf{R}^{d}\right)}
$$

and

$$
\left\|\mathcal{M}_{\Phi} f\right\|_{L^{p}\left(\mathbf{R}^{d}\right)} \leq C_{p}\|f\|_{L^{p}\left(\mathbf{R}^{d}\right)}
$$

hold for all $1<p<\infty$ and $f \in L^{p}\left(\mathbf{R}^{d}\right)$.

TheOREM 1.3. Let $\Omega$ and $T_{\Phi}^{*}$ be given as in (1.3)-(1.4). Assume that:

(i) $\Phi$ is of finite type at 0 ;

(ii) $\Omega \in B_{q}^{0,0}\left(\mathbf{S}^{n-1}\right)$ for some $q>1$.

Then for $1<p<\infty$ there exists a constant $C_{p}>0$ such that

$$
\left\|T_{\Phi}^{*} f\right\|_{L^{p}\left(\mathbf{R}^{d}\right)} \leq C_{p}\|f\|_{L^{p}\left(\mathbf{R}^{d}\right)}
$$

for every $f \in L^{p}\left(\mathbf{R}^{d}\right)$.

2. Preliminaries. Let us begin with the definition of block functions on $\mathbf{S}^{n-1}$.

Definition 2.1. (1) For $x_{0}^{\prime} \in \mathbf{S}^{n-1}$ and $0<\theta_{0} \leq 2$, the set

$$
B\left(x_{0}^{\prime}, \theta_{0}\right)=\left\{x^{\prime} \in \mathbf{S}^{n-1}:\left|x^{\prime}-x_{0}^{\prime}\right|<\theta_{0}\right\}
$$

is called a cap on $\mathbf{S}^{n-1}$.

(2) For $1<q \leq \infty$, a measurable function $b$ is called a $q$-block on $\mathbf{S}^{n-1}$ if $b$ is a function supported on some cap $I=B\left(x_{0}^{\prime}, \theta_{0}\right)$ with $\|b\|_{L^{q}} \leq|I|^{-1 / q^{\prime}}$ where $|I|=\sigma(I)$ and $1 / q+1 / q^{\prime}=1$.

(3) $B_{q}^{\kappa, v}\left(\mathbf{S}^{n-1}\right)=\left\{\Omega \in L^{1}\left(\mathbf{S}^{n-1}\right): \Omega=\sum_{\mu=1}^{\infty} c_{\mu} b_{\mu}\right.$ where each $c_{\mu}$ is a complex number; each $b_{\mu}$ is a q-block supported on a cap $I_{\mu}$ on $\mathbf{S}^{n-1}$; and $M_{q}^{\kappa, v}\left(\left\{c_{k}\right\},\left\{I_{k}\right\}\right)$ $\left.=\sum_{\mu=1}^{\infty}\left|c_{\mu}\right|\left(1+\phi_{\kappa, v}\left(\left|I_{\mu}\right|\right)\right)<\infty\right\}$, where

$$
\phi_{\kappa, v}(t)=\chi_{(0,1)}(t) \int_{t}^{1} u^{-1-\kappa} \log ^{v}\left(u^{-1}\right) d u .
$$


One observes that

$$
\begin{aligned}
& \phi_{\kappa, v}(t) \sim t^{-\kappa} \log ^{v}\left(t^{-1}\right) \text { as } t \rightarrow 0 \text { for } \kappa>0, v \in \mathbf{R} \\
& \phi_{0, v}(t) \sim \log ^{v+1}\left(t^{-1}\right) \text { as } t \rightarrow 0 \text { for } v>-1
\end{aligned}
$$

The following properties of $B_{q}^{\kappa, v}$ can be found in [KS]:

(i) $B_{q}^{\kappa, v_{2}} \subset B_{q}^{\kappa, v_{1}}$ if $v_{2}>v_{1}>-1$ and $\kappa \geq 0$;

(ii) $B_{q}^{\kappa_{2}, v_{2}} \subset B_{q}^{\kappa_{1}, v_{1}}$ if $v_{1}, v_{2}>-1$ and $0 \leq \kappa_{1}<\kappa_{2}$;

(iii) $B_{q_{2}}^{\kappa, v} \subset B_{q_{1}}^{\kappa, v}$ if $1<q_{1}<q_{2}$;

(iv) $L^{q}\left(\mathbf{S}^{n-1}\right) \subset B_{q}^{\kappa, v}\left(\mathbf{S}^{n-1}\right)$ for $v>-1$ and $\kappa \geq 0$.

In their investigations of block spaces, Keitoku and Sato showed in [KS] that these spaces enjoy the following properties:

LEMmA 2.2. (i) If $1<p \leq q \leq \infty$, then for $\kappa>\frac{1}{p^{\prime}}$ we have

$$
B_{q}^{\kappa, v}\left(\mathbf{S}^{n-1}\right) \subseteq L^{p}\left(\mathbf{S}^{n-1}\right) \text { for any } v>-1 ;
$$

(ii)

$$
B_{q}^{\kappa, v}\left(\mathbf{S}^{n-1}\right)=L^{q}\left(\mathbf{S}^{n-1}\right) \text { if and only if } \kappa \geq \frac{1}{q^{\prime}} \text { and } v \geq 0
$$

(iii) for any $v>-1$, we have

$$
\bigcup_{q>1} B_{q}^{0, v}\left(\mathbf{S}^{n-1}\right) \nsubseteq \bigcup_{q>1} L^{q}\left(\mathbf{S}^{n-1}\right) .
$$

For a $q$-block function $b$ on $\mathbf{S}^{n-1}$ supported in an interval with $q>1$ and $\|b\|_{q} \leq$ $|I|^{-1 / q^{\prime}}, 1 / q+1 / q^{\prime}=1$, we define the function $\tilde{b}$ on $\mathbf{S}^{n-1}$ by

$$
\tilde{b}(x)=b(x)-\int_{\mathbf{S}^{n-1}} b(u) d \sigma(u) .
$$

Then one can easily see that $\tilde{b}$ enjoys the following properties:

$$
\begin{aligned}
\int_{\mathbf{S}^{n-1}} \tilde{b}(u) d \sigma(u) & =0 \\
\|\tilde{b}\|_{q} & \leq 2|I|^{-1 / q^{\prime}} \\
\|\tilde{b}\|_{1} & \leq 2 .
\end{aligned}
$$

To simplify matters, we shall call the function $\tilde{b}$ the blocklike function corresponding to the block function $b$.

We shall need the following two lemmas from [FGP].

LEMMA 2.3. Let $\Phi: B(0,1) \rightarrow \mathbf{R}^{d}$ be a smooth mapping and $\Omega$ be a homogeneous function of degree 0 . Suppose that $\Phi$ is of finite type at 0 and $\Omega \in L^{q}\left(\mathbf{S}^{n-1}\right)$ for some $q>1$. Then there are $N \in \mathbf{N}, \delta \in(0,1], C>0$ and $j_{0} \in \mathbf{Z}_{-}$such that

$$
\left|\int_{2^{j-1} \leq|y|<2^{j}} e^{-i \xi \cdot \Phi(y)} \frac{\Omega(y)}{|y|^{n}} d y\right| \leq C\|\Omega\|_{q}\left(2^{N j}|\xi|\right)^{-\delta}
$$


for all $j \leq j_{0}$ and $\xi \in \mathbf{R}^{d}$.

Lemma 2.4. Let $m \in \mathbf{N}$ and $R(\cdot)$ be a real-valued polynomial on $\mathbf{R}^{n}$ with $\operatorname{deg}(R) \leq m-1$. Suppose that

$$
P(y)=\sum_{|\alpha|=m} a_{\alpha} y^{\alpha}+R(y)
$$

$\Omega$ is a homogeneous function of degree zero, and $\Omega \in L^{q}\left(\mathbf{S}^{n-1}\right)$ for some $q>1$. Then there exists a constant $C=C(m, n)>0$ such that

$$
\left|\int_{2^{j-1} \leq|y|<2^{j}} e^{-i P(y)} \frac{\Omega(y)}{|y|^{n}} d y\right| \leq C\|\Omega\|_{q}\left(2^{m j} \sum_{|\alpha|=m}\left|a_{\alpha}\right|\right)^{-\frac{1}{2 q m}}
$$

holds for all $j \in \mathbf{Z}$ and $a_{\alpha} \in \mathbf{R}$.

The proofs of our results will rely heavily on the following lemma from $[\mathrm{AqP}]$ which is an extension of earlier results of Duoandikoetxea-Rubio de Francia in [DR] and Fan-Pan in $[\mathrm{FP}]$.

Lemma 2.5. Let $N \in \mathbf{N}$ and $\left\{\sigma_{k}^{(l)}: k \in \mathbf{Z}, 0 \leq l \leq N\right\}$ be a family of Borel measures on $\mathbf{R}^{n}$ with $\sigma_{k}^{(0)}=0$ for every $k \in \mathbf{Z}$. Let $\left\{a_{l}: 1 \leq l \leq N\right\} \subseteq \mathbf{R}^{+} /(0,2)$, $\left\{m_{l}: 1 \leq l \leq N\right\} \subseteq \mathbf{N},\left\{\alpha_{l}: 1 \leq l \leq N\right\} \subseteq \mathbf{R}^{+}$, and let $L_{l}: \mathbf{R}^{n} \rightarrow \mathbf{R}^{m_{l}}$ be linear transformations for $1 \leq l \leq N$. Suppose that for all $k \in \mathbf{Z}, 1 \leq l \leq N$, for all $\xi \in \mathbf{R}^{n}$ and for some $C>0, A>1, p_{0} \in(2, \infty)$ we have the following:

(i) $\left\|\sigma_{k}^{(l)}\right\| \leq C A$;

(ii) $\left|\hat{\sigma}_{k}^{(l)}(\xi)\right| \leq C A\left|a_{l}^{k A} L_{l}(\xi)\right|^{-\frac{\alpha_{l}}{A}}$;

(iii) $\left|\hat{\sigma}_{k}^{(l)}(\xi)-\hat{\sigma}_{k}^{(l-1)}(\xi)\right| \leq C A\left|a_{l}^{k A} L_{l}(\xi)\right|^{\frac{\alpha_{l}}{A}} ;$

$(i v)$

$$
\left\|\left(\sum_{k \in \mathbf{Z}}\left|\sigma_{k}^{(l)} * g_{k}\right|^{2}\right)^{\frac{1}{2}}\right\|_{p_{0}} \leq C A\left\|\left(\sum_{k \in \mathbf{Z}}\left|g_{k}\right|^{2}\right)^{\frac{1}{2}}\right\|_{p_{0}}
$$

holds for all functions $\left\{g_{k}\right\}$ on $\mathbf{R}^{n}$.

Then for $p_{0}^{\prime}<p<p_{0}$ there exists a positive constant $C_{p}$ such that

$$
\begin{array}{r}
\left\|\sum_{k \in \mathbf{Z}} \sigma_{k}^{(N)} * f\right\|_{L^{p}\left(\mathbf{R}^{n}\right)} \leq C_{p} A\|f\|_{L^{p}\left(\mathbf{R}^{n}\right)} \\
\left\|\left(\sum_{k \in \mathbf{Z}}\left|\sigma_{k}^{(N)} * f\right|^{2}\right)^{\frac{1}{2}}\right\|_{L^{p}\left(\mathbf{R}^{n}\right)} \leq C_{p} A\|f\|_{L^{p}\left(\mathbf{R}^{n}\right)}
\end{array}
$$

hold for all $f$ in $L^{p}\left(\mathbf{R}^{n}\right)$. The constant $C_{p}$ is independent of the linear transformations $\left\{L_{l}\right\}_{l=1}^{N}$.

We shall also need the following result from $[\mathrm{DR}]$ (see also $[\mathrm{AqP}]$ ):

Lemma 2.6. Let $\left\{\lambda_{j}: j \in \mathbf{Z}\right\}$ be a sequence of Borel measures in $\mathbf{R}^{n}$ and let 
$\lambda^{*}(f)=\sup _{j \in \mathbf{Z}}|| \lambda_{j}|* f|$. Assume that

$$
\left\|\lambda^{*}(f)\right\|_{q} \leq B\|f\|_{q} \text { for some } q>1 \text { and } B>1
$$

Then, for arbitrary functions $\left\{g_{j}\right\}$ on $\mathbf{R}^{n}$ and $\left|\frac{1}{p_{0}}-\frac{1}{2}\right|=\frac{1}{2 q}$, the following inequality holds

$$
\left\|\left(\sum_{k \in \mathbf{Z}}\left|\lambda_{k} * g_{k}\right|^{2}\right)^{\frac{1}{2}}\right\|_{p_{0}} \leq\left(B \sup _{k \in \mathbf{Z}}\left\|\lambda_{k}\right\|\right)^{\frac{1}{2}}\left\|\left(\sum_{k \in \mathbf{Z}}\left|g_{k}\right|^{2}\right)^{\frac{1}{2}}\right\|_{p_{0}} .
$$

3. $L^{p}$ boundedness of certain maximal functions. For given sequences $\left\{\mu_{k}\right\}_{k \in \mathbf{Z}}$ and $\left\{\tau_{k}\right\}_{k \in \mathbf{Z}}$ of nonnegative Borel measures on $\mathbf{R}^{n}$ we define the maximal functions $\mu^{*}$ and $\tau^{*}$ by

$$
\mu^{*}(f)=\sup _{k \in \mathbf{Z}}\left|\mu_{k} * f\right| \text { and } \tau^{*}(f)=\sup _{k \in \mathbf{Z}}\left|\tau_{k} * f\right| .
$$

We have the following lemma.

Lemma 3.1. Let $\left\{\mu_{k}\right\}_{k \in \mathbf{Z}}$ and $\left\{\tau_{k}\right\}_{k \in \mathbf{Z}}$ be sequences of nonnegative Borel measures on $\mathbf{R}^{n}$. Let $L: \mathbf{R}^{n} \rightarrow \mathbf{R}^{m}$ be a linear transformation. Suppose that for all $k \in \mathbf{Z}, \xi \in \mathbf{R}^{n}$, for some $a \geq 2, \alpha, C>0$ and for some constant $B>1$ we have

(i) $\left\|\mu_{k}\right\| \leq B ;\left\|\tau_{k}\right\| \leq B$

(ii) $\left|\hat{\mu}_{k}(\xi)\right| \leq C B\left(a^{k B}|L(\xi)|\right)^{-\frac{\alpha}{B}}$;

(iii) $\left|\hat{\mu}_{k}(\xi)-\hat{\tau}_{k}(\xi)\right| \leq C B\left(a^{k B}|L(\xi)|\right)^{\frac{\alpha}{B}}$;

$(i v)$

$$
\left\|\tau^{*}(f)\right\|_{p} \leq B\|f\|_{p} \text { for all } 1<p \leq \infty \text { and } f \in L^{p}\left(\mathbf{R}^{n}\right) .
$$

Then the inequality

$$
\left\|\mu^{*}(f)\right\|_{p} \leq C_{p} B\|f\|_{p}
$$

holds for all $1<p \leq \infty$ and $f$ in $L^{p}\left(\mathbf{R}^{n}\right)$ with a constant $C_{p}$ independent of $B$ and $L$.

Proof. By the arguments in the proof of Lemma 6.2 in $[\mathrm{FP}]$, we may assume that $m \leq n$ and $L \xi=\pi_{m}^{n} \xi=\left(\xi_{1}, \ldots, \xi_{m}\right)$ for $\xi=\left(\xi_{1}, \ldots, \xi_{n}\right)$. Now, choose and fix a $\theta \in \mathcal{S}\left(\mathbf{R}^{m}\right)$ such that $\hat{\theta}(\xi)=1$ for $|\xi| \leq 1$ and $\hat{\theta}(\xi)=0$ for $|\xi| \geq 2$. For each $k \in \mathbf{Z}$, let $\left(\theta_{k}\right) \hat{(}(\xi)=\hat{\theta}\left(a^{k B} \xi\right)$, and define the sequence of measures $\left\{\Upsilon_{k}\right\}$ by

$$
\hat{\Upsilon}_{k}(\xi)=\hat{\mu}_{k}(\xi)-\left(\theta_{k} \hat{)}\left(\pi_{m}^{n} \xi\right) \hat{\tau}_{k}(\xi)\right.
$$

By (i)-(iii) we get

$$
\left|\hat{\Upsilon}_{k}(\xi)\right| \leq C B\left(a^{k B}\left|\pi_{m}^{n} \xi\right|\right)^{ \pm \frac{\alpha}{B}}
$$

for $\xi \in \mathbf{R}^{n}$. Let

$$
S_{\Upsilon}(f)(x)=\left(\sum_{k \in \mathbf{Z}}\left|\Upsilon_{k} * f(x)\right|^{2}\right)^{\frac{1}{2}} \text { and } \Upsilon^{*}(f)=\sup _{k \in \mathbf{Z}}|| \Upsilon_{k}|* f|
$$


Then by using (3.3) we have

$$
\begin{aligned}
& \mu^{*}(f)(x) \leq S_{\Upsilon}(f)(x)+C\left(\mathcal{M}_{\mathbf{R}^{m}} \otimes i d_{\mathbf{R}^{n-m}}\right)\left(\tau^{*}(f)(x)\right) \\
& \Upsilon^{*}(f)(x) \leq S_{\Upsilon}(f)(x)+2 C\left[\left(\mathcal{M}_{\mathbf{R}^{m}} \otimes i d_{\mathbf{R}^{n-m}}\right)\right]\left(\tau^{*}(f)(x)\right)
\end{aligned}
$$

where $\mathcal{M}_{\mathbf{R}^{d}}$ is the classical Hardy-Littlewood maximal function on $\mathbf{R}^{d}$.

By (3.4) and Plancherel's theorem we obtain

$$
\left\|S_{\Upsilon}(f)\right\|_{2} \leq C B\|f\|_{2}
$$

which when combined with the $L^{p}$ boundedness of $\mathcal{M}_{\mathbf{R}^{d}}$, (3.1), and (3.6)-(3.7) gives that

$$
\left\|\Upsilon^{*}(f)\right\|_{2} \leq C B\|f\|_{2}
$$

with $C$ independent of $B$. By using the fact $\left\|\Upsilon_{k}\right\| \leq C B$ together with Lemma 2.6 (for $q=2$ ) we get

$$
\|\left(\sum_{k \in \mathbf{Z}}\left(\left|\Upsilon_{k} * g_{k}\right|^{2}\right)^{\frac{1}{2}}\left\|_{p_{0}} \leq C_{p_{0}} B\right\|\left(\sum_{k \in \mathbf{Z}}\left|g_{k}\right|^{2}\right)^{\frac{1}{2}} \|_{p_{0}}\right.
$$

if $1 / 4=\left|1 / p_{0}-1 / 2\right|$. Now, by (3.4), (3.9) and applying Lemma 2.5 we get

$$
\left\|S_{\Upsilon}(f)\right\|_{p} \leq C_{p} B\|f\|_{p} \text { for } p \in\left(\frac{4}{3}, 4\right) .
$$

Again, the $L^{p}$ boundedness of $\mathcal{M}_{\mathbf{R}^{d}},(3.1),(3.6)$ and (3.10) imply that

$$
\left\|\Upsilon^{*}(f)\right\|_{p} \leq C B\|f\|_{p} \text { for } p \in\left(\frac{4}{3}, 4\right) .
$$

Reasoning as above, (3.4), (3.11), Lemma 2.5 and Lemma 2.6 provide

$$
\left\|S_{\Upsilon}(f)\right\|_{p} \leq C_{p} B\|f\|_{p} \text { for } p \in\left(\frac{8}{7}, 8\right)
$$

By successive application of the above argument we ultimately obtain that

$$
\left\|S_{\Upsilon}(f)\right\|_{p} \leq C_{p} B\|f\|_{p} \text { for } p \in(1, \infty) .
$$

Therefore, by the $L^{p}$ boundedness of $\mathcal{M}_{\mathbf{R}^{d}},(3.1),(3.5)$ and (3.13) we conclude that

$$
\left\|\mu^{*}(f)\right\|_{p} \leq C_{p} B\|f\|_{p} \text { for } p \in(1, \infty) .
$$

Finally, the inequality (3.2) holds trivially for $p=\infty$. This concludes the proof of our lemma.

Definition 3.2. Let $\tilde{b}(\cdot)$ be a blocklike function defined as in (2.2) and $\Gamma$ be an arbitrary function on $\mathbf{R}^{n}$. Define the measures $\left\{\sigma_{\Gamma, \tilde{b}, j}: j \in \mathbf{Z}\right\}$ and the maximal operator $\sigma_{\Gamma, \tilde{b}}^{*}$ on $\mathbf{R}^{n}$ by

$$
\begin{aligned}
\int_{\mathbf{R}^{d}} f d \sigma_{\Gamma, \tilde{b}, j} & =\int_{2^{j-1} \leq|u|<2^{j}} f(\Gamma(u)) \frac{\tilde{b}(u)}{|u|^{n}} d u ; \\
\sigma_{\Gamma, \tilde{b}}^{*}(f) & =\sup _{j \in \mathbf{Z}}|| \sigma_{\Gamma, \tilde{b}, j}|* f| .
\end{aligned}
$$


These measures will be useful only in the case $|I| \geq e^{-2}$ where $I$ is the support of $b$. On the other hand, for the case $|I|<e^{-2}$ we need to define the following measures.

DeFINITION 3.3. Let $\tilde{b}(\cdot)$ be a q-blocklike function defined as in (2.2) and $\Gamma$ be an arbitrary function on $\mathbf{R}^{n}$. We define the measures $\left\{\lambda_{\Gamma, \tilde{b}, j}: j \in \mathbf{Z}\right\}$ and the maximal operators $\lambda_{\Gamma, \tilde{b}}^{*}$ on $\mathbf{R}^{n}$ by

$$
\begin{aligned}
\int_{\mathbf{R}^{d}} f d \lambda_{\Gamma, \tilde{b}, j} & =\int_{\omega^{j-1} \leq|u|<\omega^{j}} f(\Gamma(u)) \frac{\tilde{b}(u)}{|u|^{n}} d u ; \\
\lambda_{\Gamma, \tilde{b}}^{*} f(x) & =\sup _{j \in \mathbf{Z}}|| \lambda_{\Gamma, \tilde{b}, j}|* f(x)|
\end{aligned}
$$

where $\omega=2^{\left[\log \left(|I|^{-1}\right)\right]},|I|<e^{-2}$ and $[\cdot]$ denotes the greatest integer function.

Lemma 3.4. Let $\Phi: B(0,1) \rightarrow \mathbf{R}^{d}$ be a smooth mapping and for $q>1$ let $\tilde{b}$ be a q-blocklike function defined as in (2.2). Suppose that $\Phi$ is of finite type at 0 . If $|I|<e^{-2}$, then there are $N \in \mathbf{N}, \delta \in(0,1], C>0$ and $j_{0} \in \mathbf{Z}_{-}$such that

$$
\left|\hat{\lambda}_{\Phi, \tilde{b}, j}(\xi)\right| \leq C[\log (|I|)]\left(\omega^{N j}|\xi|\right)^{-\frac{\delta}{\left[\log \left(|I|^{-1}\right)\right]}}
$$

for all $j \leq j_{0}, \xi \in \mathbf{R}^{d}$ with $C$ independent of $j$ and $\left[\log \left(|I|^{-1}\right)\right]$.

Proof. By (2.4), Lemma 2.3 and the definition of $\lambda_{\Phi, \tilde{b}, j}$ we get

$$
\begin{aligned}
\left|\hat{\lambda}_{\Phi, \tilde{b}, j}(\xi)\right| & \leq \sum_{s=0}^{\left[\log \left(|I|^{-1}\right)\right]-1}\left|\int_{\omega^{(j-1)} 2^{s} \leq|y|<\omega^{(j-1)} 2^{(s+1)}} e^{-i \xi \cdot \Phi(y)} \frac{\tilde{b}(y)}{|y|^{n}} d y\right| \\
& \leq \sum_{s=0}^{\left[\log \left(|I|^{-1}\right)\right]-1} C|I|^{-\frac{1}{q^{\prime}}\left(\omega^{N(j-1)} 2^{N(s+1)}|\xi|\right)^{-\delta}} \\
& \leq C|I|^{-\frac{1}{q^{\prime}}} \omega^{\delta N}\left(\omega^{N j}|\xi|\right)^{-\delta}\left(\frac{1-\omega^{-\delta N}}{1-2^{\delta N}}\right) \\
& \leq C \omega^{\delta N}|I|^{-\frac{1}{q^{\prime}}}\left(\omega^{j}|\xi|\right)^{-\delta}
\end{aligned}
$$

By interpolating between this estimate and the trivial estimate

$$
\left|\hat{\lambda}_{\Phi, \tilde{b}, j}(\xi)\right| \leq C\left[\log \left(|I|^{-1}\right)\right]
$$

we get the estimate in (3.19). This concludes the proof of our lemma.

By Lemma 2.4 and the argument used in the proof of Lemma 3.4 we get the following:

Lemma 3.5. Let $m \in \mathbf{N}, \tilde{b}$ be a q-blocklike function (for $q>1$ ) defined as in (2.2) and $R(\cdot)$ be a real-valued polynomial on $\mathbf{R}^{n}$ with $\operatorname{deg}(R) \leq m-1$. Suppose

$$
P(y)=\sum_{|\alpha|=m} a_{\alpha} y^{\alpha}+R(y),
$$


and $|I|<e^{-2}$. Then there exists a constant $C=C(m, n)>0$ such that

$$
\left|\int_{\omega^{j-1} \leq|u|<\omega^{j}} e^{-i P(y)} \frac{\tilde{b}(y)}{|y|^{n}} d y\right| \leq C\left[\log \left(|I|^{-1}\right)\right]\left(\omega^{m j} \sum_{|\alpha|=m}\left|a_{\alpha}\right|\right)^{-\frac{1}{2 \dot{q} m\left[\log \left(|I|^{-1}\right)\right]}}
$$

holds for all $j \in \mathbf{Z}$ and $a_{\alpha} \in \mathbf{R}$.

By Proposition 1 on page 477 of [St] it is easy to see that the following result holds.

LEMma 3.6. Let $\mathcal{P}=\left(P_{1}, \ldots, P_{d}\right)$ be a polynomial mapping from $\mathbf{R}^{n}$ into $\mathbf{R}^{d}$. Let $\operatorname{deg}(\mathcal{P})=\max _{1 \leq j \leq d} \operatorname{deg}\left(P_{j}\right)$. Suppose that $\tilde{b}(\cdot)$ is a blocklike function defined as in (2.2) and $\sigma_{\mathcal{P}, \Omega}^{*}$ be given as in (2.16). Then for every $1<p \leq \infty$, there exists a constant $C_{p}$ independent of $\tilde{b}$ and the coefficients of $\mathcal{P}$ such that

$$
\left\|\sigma_{\mathcal{P}, \tilde{b}}^{*}(f)\right\|_{p} \leq C_{p}\|f\|_{p}
$$

for $f \in L^{p}\left(\mathbf{R}^{d}\right)$.

By the above lemma and the proof of Lemma 3.4 we obtain the following:

Lemma 3.7. Let $\mathcal{P}=\left(P_{1}, \ldots, P_{d}\right)$ be a polynomial mapping from $\mathbf{R}^{n}$ into $\mathbf{R}^{d}$ and $\tilde{b}$ be a q-blocklike function defined as in (2.2). Let $\operatorname{deg}(\mathcal{P})=\max _{1 \leq j \leq d} \operatorname{deg}\left(P_{j}\right)$. Suppose that $|I|<e^{-2}$. Then for every $1<p \leq \infty$, there exists a constant $C_{p}$ independent of $\tilde{b}$ and the coefficients of $\mathcal{P}$ such that

$$
\left\|\lambda_{\mathcal{P}, \tilde{b}}^{*}(f)\right\|_{p} \leq C_{p}\left[\log \left(|I|^{-1}\right)\right]\|f\|_{p}
$$

for $f \in L^{p}\left(\mathbf{R}^{d}\right)$.

Our next step is to prove the following result on maximal functions:

TheOrem 3.8. Let $\Phi: B(0,1) \rightarrow \mathbf{R}^{d}$ be a smooth mapping and for $q>1$ let $\tilde{b}$ be a q-blocklike function defined as in (2.2). Suppose that $\Phi$ is of finite type at 0 . Then for $1<p \leq \infty$ and $f \in L^{p}\left(\mathbf{R}^{d}\right)$ there exists a positive constant $C_{p}$ which is independent of $\tilde{b}$ such that

$$
\begin{aligned}
& \left\|\lambda_{\Phi, \tilde{b}}^{*}(f)\right\|_{L^{p}\left(\mathbf{R}^{d}\right)} \leq C_{p}\left[\log \left(|I|^{-1}\right)\right]\|f\|_{L^{p}\left(\mathbf{R}^{d}\right)} \text { if }|I|<e^{-2} \\
& \left\|\sigma_{\Phi, \tilde{b}}^{*}(f)\right\|_{L^{p}\left(\mathbf{R}^{d}\right)} \leq C_{p}\|f\|_{L^{p}\left(\mathbf{R}^{d}\right)} \text { if }|I| \geq e^{-2} .
\end{aligned}
$$

Proof. Assume first that $|I|<e^{-2}$. Without loss of generality we may assume that $\tilde{b} \geq 0$. By Lemma 3.4, there are $N \in \mathbf{N}, \delta \in(0,1], C>0$ and $k_{0} \in \mathbf{Z}_{-}$such that

$$
\left|\hat{\lambda}_{\Phi, \tilde{b}, k}(\xi)\right| \leq C\left[\log \left(|I|^{-1}\right)\right]\left(\omega^{N k}|\xi|\right)^{-\frac{\delta}{\left[\log \left(|I|^{-1}\right)\right]}}
$$


for all $k \leq k_{0}, \xi \in \mathbf{R}^{d}$ with $C$ independent of $k$ and $\left[\log \left(|I|^{-1}\right)\right]$ where $\omega=2^{\left[\log \left(|I|^{-1}\right)\right]}$. For $\Phi=\left(\Phi_{1}, \ldots, \Phi_{d}\right)$ we let $\mathcal{P}=\left(P_{1}, \ldots, P_{d}\right)$ where

$$
P_{j}(y)=\sum_{|\beta| \leq N-1} \frac{1}{\beta !} \frac{\partial^{\beta} \Phi_{j}}{\partial y^{\beta}}(0) y^{\beta}, \quad 1 \leq j \leq d .
$$

Then we have

$$
\left|\hat{\lambda}_{\Phi, \tilde{b}, k}(\xi)-\hat{\lambda}_{\mathcal{P}, \tilde{b}, k}(\xi)\right| \leq C\left[\log \left(|I|^{-1}\right)\right] \omega^{-N}\left(\omega^{N k}|\xi|\right) .
$$

By (2.5) we have

$$
\left|\hat{\lambda}_{\Phi, \tilde{b}, k}(\xi)-\hat{\lambda}_{\mathcal{P}, \tilde{b}, k}(\xi)\right| \leq C\left[\log \left(|I|^{-1}\right)\right] .
$$

By interpolating between this estimate and (3.23) we get

$$
\left|\hat{\lambda}_{\Phi, \tilde{b}, k}(\xi)-\hat{\lambda}_{\mathcal{P}, \tilde{b}, k}(\xi)\right| \leq C\left[\log \left(|I|^{-1}\right)\right]\left(\omega^{N k}|\xi|\right)^{\frac{\delta}{[\log (|I|-1)]}} .
$$

Therefore, (3.20) follows from (3.22), (3.25), Lemma 3.1 and Lemma 3.7. The proof of the inequality (3.21) will be much easier. In fact, it follows from (2.4)-(2.5), Lemma 2.3, 3.1, and 3.6. We omit the details.

4. Proofs of the theorems. By assumption, $\Omega$ can be written as $\Omega=\sum_{\mu=1}^{\infty} c_{\mu} b_{\mu}$ where $c_{\mu} \in \mathbf{C}, b_{\mu}$ is a $q$-block with support on an ia cap $I_{\mu}$ on $\mathbf{S}^{n-1}$ and

$$
M_{q}^{0,0}\left(\left\{c_{k}\right\},\left\{I_{k}\right\}\right)=\sum_{\mu=1}^{\infty}\left|c_{\mu}\right|\left(1+\left(\log \left|I_{\mu}\right|^{-1}\right)\right)<\infty .
$$

For each $\mu=1,2, \ldots$, let $\tilde{b}_{\mu}$ be the blocklike function corresponding to $b_{\mu}$. By the vanishing condition on $\Omega$ we have

$$
\Omega=\sum_{\mu=1}^{\infty} c_{\mu} \tilde{b}_{\mu}
$$

and hence

$$
\left\|T_{\Phi} f\right\|_{p} \leq \sum_{\mu=1}^{\infty}\left|c_{\mu}\right|\left\|T_{\Phi, \tilde{b}_{\mu}} f\right\|_{p}
$$

where

$$
T_{\Phi, \tilde{b}_{\mu}} f(x)=\text { p.v. } \int_{B(0,1)} f(x-\Phi(u)) \frac{\tilde{b}_{\mu}\left(u^{\prime}\right)}{|u|^{n}} d u .
$$

Let $\delta, N, \mathcal{P}$ be given as in the proof of Theorem 3.8. For $1 \leq j \leq d$, let $a_{j, \beta}=$ $\frac{1}{\beta !} \frac{\partial^{\beta} \Phi_{j}}{\partial y^{\beta}}(0)$. For $0 \leq l \leq N-1$ we define $Q^{l}=\left(Q_{1}^{l}, \ldots, Q_{d}^{l}\right)$ by

$$
Q_{j}^{l}(y)=\sum_{|\beta| \leq l} a_{j, \beta} y^{\beta}, \quad j=1, \ldots, d
$$


when $0 \leq l \leq N-1$ and $Q^{N}=\Phi$. For each $0 \leq l \leq N$, let $\lambda_{\tilde{b}_{\mu}, k}^{(l)}=\lambda_{Q^{l}, \tilde{b}_{\mu}, k}$ and $\sigma_{\tilde{b}_{\mu}, k}^{(l)}$ $=\sigma_{Q^{l}, \tilde{b}_{\mu}, k}$. Then by (2.3)-(2.5), Lemma 2.4 we have

$$
\begin{aligned}
\| \sigma_{\tilde{b}_{\mu}, k}^{(l)} \mid & \leq C ; \\
\left|\hat{\sigma}_{\tilde{b}_{\mu}, k}^{(l)}(\xi)\right| & \leq C\left(2^{l k} \sum_{|\beta|=l}\left|\sum_{j=l}^{d} a_{j, \beta} \xi_{j}\right|\right)^{-\frac{1}{2 q^{\prime} l}} ; \\
\left|\hat{\sigma}_{\tilde{b}_{\mu}, k}^{(N)}(\xi)-\hat{\sigma}_{\tilde{b}_{\mu}, k}^{(N-1)}(\xi)\right| & \leq C\left(2^{N k}|\xi|\right) ; \\
\left|\hat{\sigma}_{\tilde{b}_{\mu}, k}^{(l)}(\xi)-\hat{\sigma}_{\tilde{b}_{\mu}, k}^{(l-1)}(\xi)\right| & \leq C\left(2^{l k} \sum_{|\beta|=l}\left|\sum_{j=l}^{d} a_{j, \beta} \xi_{j}\right|\right)
\end{aligned}
$$

for $\left|I_{\mu}\right| \geq e^{-2}, \mu=1,2, \ldots, 0 \leq l \leq N-1$, and $k \leq k_{0}$. Also, by (2.3)-(2.5), Lemma 3.5 , and the same argument as in the proof (3.25) we have

$$
\begin{aligned}
\left\|\lambda_{\tilde{b}_{\mu}, k}^{(l)}\right\| & \leq C A_{\mu} ; \\
\left|\hat{\lambda}_{\tilde{b}_{\mu}, k}^{(l)}(\xi)\right| & \leq C A_{\mu}\left(2^{l A_{\mu} k} \sum_{|\beta|=l}\left|\sum_{j=l}^{d} a_{j, \beta} \xi_{j}\right|\right)^{-\frac{1}{A_{\mu} 2 q^{\prime} l}} ; \\
\left|\hat{\lambda}_{\tilde{b}_{\mu}, k}^{(l)}(\xi)-\hat{\lambda}_{\tilde{b}_{\mu}, k}^{(l-1)}(\xi)\right| & \leq C A_{\mu}\left(2^{l A_{\mu} k} \sum_{|\beta|=l}\left|\sum_{j=l}^{d} a_{j, \beta} \xi_{j}\right|\right)^{\frac{1}{A_{\mu} 2 q^{\prime} l}}
\end{aligned}
$$

where $A_{\mu}=\left[\log \left(\left|I_{\mu}\right|^{-1}\right)\right],\left|I_{\mu}\right|<e^{-2}, \mu=1,2, \ldots, k \leq k_{0}, 0 \leq l \leq N-1$.

By (3.20)-(3.22), (3.25), (4.5)-(4.11), Theorem 3.8, Lemmas 2.5-2.6, and 3.6-3.7 we get

$$
\begin{aligned}
\left\|T_{\Phi, \tilde{b}_{\mu}} f\right\|_{p} & =\left\|\sum_{j \in \mathbf{Z}_{-}} \lambda_{\tilde{b}_{\mu}, k}^{(N)} * f\right\|_{p} \leq C_{p} A_{\mu}\|f\|_{p} \text { if }\left|I_{\mu}\right|<e^{-2} ; \\
\left\|T_{\Phi, \tilde{b}_{\mu}} f\right\|_{p} & =\left\|\sum_{j \in \mathbf{Z}_{-}} \sigma_{\tilde{b}_{\mu}, k}^{(N)} * f\right\|_{p} \leq C_{p}\|f\|_{p} \text { if }\left|I_{\mu}\right| \geq e^{-2}
\end{aligned}
$$

for every $f \in L^{p}\left(\mathbf{R}^{d}\right), \mu=1,2, \ldots$, and for all $p, 1<p<\infty$. Hence, (1.7) follows from (4.1), (4.3) and (4.12)-(4.13). On the other hand, (1.8) follows from (3.20)-(3.21), (4.2) and the following inequality

$$
\begin{gathered}
\mathcal{M}_{\Phi} f(x) \leq 4 \sum_{\mu=1}^{\infty}\left|c_{\mu}\right| \sigma_{\Phi, \tilde{b}_{\mu}}^{*}(|f|)(x) \\
\leq 4 \sum_{\mu=1,\left|I_{\mu}\right| \geq e^{-2}}^{\infty}\left|c_{\mu}\right| \sigma_{\Phi, \tilde{b}_{\mu}}^{*}(|f|)(x)+8 \sum_{\mu=1,\left|I_{\mu}\right|<e^{-2}}^{\infty}\left|c_{\mu}\right| \lambda_{\Phi, \tilde{b}_{\mu}}^{*}(|f|)(x) .
\end{gathered}
$$

This concludes the proof of Theorem 1.2.

Finally, the proof of Theorem 1.3 follows from the above estimates and the techniques in $[\mathrm{AqP}]$. We omit the details. 


\section{REFERENCES}

[AqAsP] H. Al-QASSEm, A. Al-SAlman And Y. PAn, Singular integrals associated to homogeneous mappings with rough kernels, Hokkaido Math. J., 33(2004), pp. 551-569.

[AqP] H. AL-QASSEM AND Y. PAN, $L^{p}$ estimates for singular integrals with kernels belonging to certain block spaces, Revista Matemática Iberoamericana, (18) 3 (2002), pp. 701-730.

[DR] J. Duonndikoetxea And J. L. Rubio De Francia, Maximal functions and singular integral operators via Fourier transform estimates, Invent. Math., 84(1986), pp. 541561.

[FGP] D. FAN, K. GUO, AND Y. PAN, Singular integrals along submanifolds of finite type, Mich. Math. J., 45(1998), pp. 135-142.

[FP] D. FAN AND Y. PAN, Singular integral operators with rough kernels supported by subvarieties, Amer J. Math., 119(1997), pp. 799-839.

[KS] M. Keitoku And E. Sato, Block spaces on the unit sphere in $\mathbf{R}^{n}$, Proc. Amer. Math. Soc., 119(1993), pp. 453-455.

[LTW] S. Lu, M. Taibleson And G. Weiss, Spaces Generated by Blocks, Beijing Normal University Press, 1989, Beijing.

[So] F. SoRIA, Characterizations of classes of functions generated by blocks and associated Hardy spaces, Ind. Univ. Math. J., 34:3(1985), pp. 463-492.

[St] E. M. SteIn, Problems in harmonic analysis related to curvature and oscillatory integrals, Proc. Int l. Cong. Math., (1986), pp. 196-221. 
H. AL-QASSEM, A. AL-SALMAN AND Y. PAN 